\title{
On HT-convexity and Hadamard-type inequalities
}

\author{
Shu-Ping Bai ${ }^{1}$, Shu-Hong Wang ${ }^{1}$ (I) and Feng $\mathrm{Qi}^{2,33^{*}}$ (D)
}

"Correspondence:

qifeng618@hotmail.com

${ }^{2}$ Institute of Mathematics, Henan

Polytechnic University, Jiaozuo,

China

${ }^{3}$ School of Mathematical Sciences,

Tianjin Polytechnic University,

Tianjin, China

Full list of author information is

available at the end of the article sharing, adaptation, distribution and reproduction in any medium or format, as long as you give appropriate credit to the original author(s) and the source, provide a link to the Creative Commons licence, and indicate if changes were made. The images or other third party material in this article are included in the article's Creative Commons licence, unless indicated otherwise in a credit line to the material. If material is not included in the article's Creative Commons licence and your intended use is not permitted by statutory regulation or exceeds the permitted use, you will need to obtain permission directly from the copyright holder. To view a copy of this licence, visit http://creativecommons.org/licenses/by/4.0/.

\section{Introduction}

The classical convexity and concavity of functions are two fundamental notions in mathematics. Mathematicians and scientists can see them in research papers, monographs, and textbooks devoted to the theory of convex analysis [13]. The origin of the theory of convex functions is generally attributed to Jensen [10], although he was not the first person to deal with such functions. Among Jensen's predecessors, there were Hermite [6], Hölder [7], and Stolz [19], to name a few. The well-known book [5] played an indispensable role in the popularization of the theory of convex functions.

The famous Hermite-Hadamard integral inequality in Theorem 2.1 for convex functions below is the first fundamental conclusion for convex functions and has been attracting a lot of interest from mathematicians and other scientists. In recent years, the Hermite-Hadamard integral inequality has been the subject of very active research. Various improvements, generalizations, and variants of this inequality can be found in the papers $[1,3,8,9,11,12,14,16,20,23,24,27,28,32]$ and closely related references therein. In [15], the late Pachpatte established some Hadamard-type inequalities for the product of two convex functions. Alternative Hadamard-type inequalities for the product of two convex functions were also established in the papers [25, 30, 31]. In [22], the HermiteHadamard inequality was applied to generalize and refine Young's integral inequality in terms of higher order derivatives.

Nowadays, many mathematicians have devoted their efforts to generalizations, refinements, counterparts, and extensions of the convexity of functions for adapting to other 
geometries of the domain and/or for disclosing other laws of comparison of mathematical means. In $[1,4,8]$, by replacing the weighted arithmetic means with the weighted harmonic means, the notion of HA-convex functions was introduced. In [21], Tunç and Yildrim defined the MT-convexity and obtained some new Hadamard-type inequalities for MT-convex functions. The theory of various classes of convex functions is similar to that of classical convex functions. Some inequalities are easier to state using these new convex functions and some are easier to state using the classical convex functions. In many cases the inequalities based on these new convexity notions are better than direct applications of inequalities of the classical convexity. Consequently, there is a strong interest in investigating different classes of convex functions.

In Sect. 2, we will mathematically and technically quote some definitions of several classes of convex functions and some inequalities of Hadamard-type. In Sect. 3, by using the weighted arithmetic and harmonic means, we will introduce the notion of "HTconvex functions", which is a generalization of the HA-convex functions defined in $[1,4,8]$. In Sect. 4, we will present some Hadamard-type inequalities for HT-convex functions and for the product of two HT-convex functions. In Sect. 5, we will apply newly-established inequalities to derive some inequalities for the arithmetic mean and the $p$-logarithmic mean.

\section{Preliminaries}

In this section, we will mathematically quote some preliminary notations, definitions, and known results about several classes of convex functions and the Hadamard-type inequalities.

Definition 2.1 ([2]) A function $Q$ defined on a nonempty interval $J \subseteq \mathbb{R}$ is said to be convex if

$$
Q(\lambda \tau+(1-\lambda) \mu) \leq \lambda Q(\tau)+(1-\lambda) Q(\mu)
$$

is true for all $\tau, \mu \in J$ and $\lambda \in[0,1]$.

Theorem 2.1 ([17]) Let $Q$ be a convex function defined on a nonempty interval $J \subseteq \mathbb{R}$ and $\tau, \mu \in J$ with $\tau<\mu$. Then

$$
Q\left(\frac{\tau+\mu}{2}\right) \leq \frac{1}{\mu-\tau} \int_{\tau}^{\mu} Q(x) \mathrm{d} x \leq \frac{Q(\tau)+Q(\mu)}{2} .
$$

If $Q$ is concave, the above double inequality holds reversed.

In the mathematics community, the double inequality (1) is known as the HermiteHadamard integral inequality.

Theorem 2.2 ([15, Theorem 1]) Suppose that $P, Q$ are two positive and convex functions on a nonempty interval $J \subseteq \mathbb{R}, \tau, \mu \in J$ with $\tau<\mu$, and the product of $P$ and $Q$ is Lebesgue 
integrable on $[\tau, \mu]$. Then

$$
\begin{aligned}
& 2 P\left(\frac{\tau+\mu}{2}\right) Q\left(\frac{\tau+\mu}{2}\right)-\frac{1}{6} M(\tau, \mu)-\frac{1}{3} N(\tau, \mu) \\
& \quad \leq \frac{1}{\mu-\tau} \int_{\tau}^{\mu} P(x) Q(x) \mathrm{d} x \leq \frac{1}{3} M(\tau, \mu)+\frac{1}{6} N(\tau, \mu),
\end{aligned}
$$

where

$$
M(\tau, \mu)=P(\tau) Q(\tau)+P(\mu) Q(\mu) \text { and } \quad N(\tau, \mu)=Q(\tau) Q(\mu)+P(\mu) Q(\tau) .
$$

Definition 2.2 $([1,4,8])$ Let $J \subseteq \mathbb{R} \backslash\{0\}$ be a nonempty interval. A function $Q: J \rightarrow \mathbb{R}$ is said to be HA-convex, denoted by $Q \in \mathrm{HA}(J)$, if the inequality

$$
Q\left(\frac{\tau \mu}{\lambda \tau+(1-\lambda) \mu}\right) \leq \lambda Q(\tau)+(1-\lambda) f(\mu)
$$

holds for all $\tau, \mu \in J$ and $\lambda \in[0,1]$.

Definition 2.3 ([21]) A nonnegative function $Q$ on a nonempty interval $J$ is said to be MT-convex, denoted by $Q \in \mathrm{MT}(J)$, if

$$
Q(\lambda \tau+(1-\lambda) \mu) \leq \frac{\sqrt{\lambda}}{2 \sqrt{1-\lambda}} Q(\tau)+\frac{\sqrt{1-\lambda}}{2 \sqrt{\lambda}} Q(\mu)
$$

is valid for all $\tau, \mu \in J$ and $\lambda \in(0,1)$.

Theorem 2.3 ([21]) Let $Q \in \mathrm{MT}(J), \tau, \mu \in J$ with $\tau<\mu$, and $Q$ be Lebesgue integrable on $[\tau, \mu]$. Then

$$
\frac{\pi}{2} Q\left(\frac{\tau+\mu}{2}\right) \leq Q(\tau)+Q(\mu), \quad Q\left(\frac{\tau+\mu}{2}\right) \leq \frac{1}{\mu-\tau} \int_{\tau}^{\mu} Q(x) \mathrm{d} x,
$$

and

$$
\frac{2}{\mu-\tau} \int_{\tau}^{\mu} v(x) Q(x) \mathrm{d} x \leq \frac{Q(\tau)+Q(\mu)}{2},
$$

where

$$
v(x)=\frac{2 \sqrt{(\mu-x)(x-\tau)}}{\mu-\tau}, \quad x \in[\tau, \mu] .
$$

Lemma 2.1 ([4]) Suppose that $J \subseteq(0, \infty)$ is a nonempty interval and $\tau, \mu \in J$ with $\tau<\mu$. Let $Q:[\tau, \mu] \rightarrow \mathbb{R}$ be Lebesgue integrable on $[\tau, \mu]$. Then, for $\lambda \in[0,1]$,

$$
\begin{aligned}
\int_{0}^{1} Q\left(\frac{\tau \mu}{(1-s) \tau+s \mu}\right) \mathrm{d} s= & (1-\lambda) \int_{0}^{1} Q\left(\frac{\tau \mu}{(1-s)[(1-\lambda) \tau+\lambda \mu]+s \mu}\right) \mathrm{d} s \\
& +\lambda \int_{0}^{1} Q\left(\frac{\tau \mu}{(1-s) \tau+s[(1-\lambda) \tau+\lambda \mu]}\right) \mathrm{d} s .
\end{aligned}
$$


Definition 2.4 ([18]) Two functions $P, Q: J \subseteq \mathbb{R} \rightarrow \mathbb{R}$ are said to be similarly ordered if

$$
[P(\tau)-P(\mu)][Q(\tau)-Q(\mu)] \geq 0, \quad \tau, \mu \in J .
$$

\section{HT-convexity}

We now define the concept of the HT-convexity and give several basic properties.

Definition 3.1 Let $J \subseteq \mathbb{R} \backslash\{0\}$ be a nonempty interval. A function $Q$ is called HT-convex on $J$, denoted by $Q \in \mathrm{HT}(J)$, if the inequality

$$
Q\left(\frac{\tau \mu}{\lambda \tau+(1-\lambda) \mu}\right) \leq \frac{\sqrt{\lambda}}{2 \sqrt{1-\lambda}} Q(\mu)+\frac{\sqrt{1-\lambda}}{2 \sqrt{\lambda}} Q(\tau)
$$

holds for all $\tau, \mu \in J$ and $\lambda \in(0,1)$. If the inequality in (4) is reversed, then $Q$ is called an HT-concave function.

Remark 3.1 Taking $\lambda=\frac{1}{2}$ in inequality (4) yields

$$
Q\left(\frac{2 \tau \mu}{\tau+\mu}\right) \leq \frac{Q(\tau)+Q(\mu)}{2}
$$

Remark 3.2 Suppose that $J \subseteq \mathbb{R} \backslash\{0\}$ is a nonempty interval and $Q: J \rightarrow \mathbb{R}$ is an HT-convex function.

1 If $J \subseteq(0, \infty)$ and $Q$ is an MT-convex and nondecreasing function, then $Q$ is HT-convex.

2 If $J \subseteq(0, \infty)$ and $Q$ is an HT-convex and nonincreasing function, then $Q$ is MT-convex.

3 If $J \subseteq(-\infty, 0)$ and $Q$ is an MT-convex and nonincreasing function, then $Q$ is HT-convex.

4. If $J \subseteq(-\infty, 0)$ and $Q$ is an HT-convex and nondecreasing function, then $Q$ is MT-convex.

Proposition 3.1 Suppose that $J \subseteq(0, \infty)$ is a nonempty interval. For $\tau, \mu \in J$ with $\tau<\mu$, if $P:\left[\frac{1}{\mu}, \frac{1}{\tau}\right] \rightarrow \mathbb{R}$ is MT-convex, then $Q:[\tau, \mu] \rightarrow \mathbb{R}, Q(t)=P\left(\frac{1}{t}\right)$, is HT-convex.

Proof Let $s, t \in[\tau, \mu]$ and $\lambda \in(0,1)$. Then

$$
\begin{aligned}
Q\left(\frac{s t}{t \lambda+(1-\lambda) t}\right) & =Q\left(\frac{1}{\lambda \frac{1}{t}+(1-\lambda) \frac{1}{s}}\right)=P\left(\lambda \frac{1}{t}+(1-\lambda) \frac{1}{s}\right) \\
& \leq \frac{\sqrt{\lambda}}{2 \sqrt{1-\lambda}} P\left(\frac{1}{t}\right)+\frac{\sqrt{1-\lambda}}{2 \sqrt{\lambda}} P\left(\frac{1}{s}\right)=\frac{\sqrt{\lambda}}{2 \sqrt{1-\lambda}} Q(t)+\frac{\sqrt{1-\lambda}}{2 \sqrt{\lambda}} Q(s),
\end{aligned}
$$

which shows that $Q$ is HT-convex on $[\tau, \mu]$.

Proposition 3.2 All nonnegative HA-convex functions are HT-convex. 
Proof Suppose that $Q$ is HA-convex. By the fact that $\lambda \leq \frac{\sqrt{\lambda}}{2 \sqrt{1-\lambda}}$ and $1-\lambda \leq \frac{\sqrt{1-\lambda}}{2 \sqrt{\lambda}}$, it is easy to obtain

$$
Q\left(\frac{\tau \mu}{\lambda \tau+(1-\lambda) \mu}\right) \leq \lambda Q(\mu)+(1-\lambda) Q(\tau) \leq \frac{\sqrt{\lambda}}{2 \sqrt{1-\lambda}} Q(\mu)+\frac{\sqrt{1-\lambda}}{2 \sqrt{\lambda}} Q(\tau),
$$

where $\tau, \mu \in J \subseteq \mathbb{R} \backslash\{0\}$ and $\lambda \in(0,1)$. This means that each HA-convex function is HTconvex.

\section{Hadamard-type inequalities for HT-convex functions}

Now we are in a position to establish some Hadamard-type inequalities for HT-convex functions.

Theorem 4.1 Suppose that $J \subseteq \mathbb{R} \backslash\{0\}$ is a nonempty interval, $Q \in \mathrm{HT}(J), \tau, \mu \in J$ with $\tau<\mu$, and $Q$ is Lebesgue integrable on $[\tau, \mu]$. Then

$$
Q\left(\frac{2 \tau \mu}{\tau+\mu}\right) \leq \frac{\tau \mu}{\mu-\tau} \int_{\tau}^{\mu} \frac{Q(x)}{x^{2}} \mathrm{~d} x \leq \frac{\pi}{4}[Q(\tau)+Q(\mu)] .
$$

Proof Because $Q$ is an HT-convex function, for $\tau, \mu \in J$ with $\tau<\mu$ and $\lambda \in(0,1)$, we have

$$
Q\left(\frac{\tau \mu}{\lambda \tau+(1-\lambda) \mu}\right) \leq \frac{\sqrt{\lambda}}{2 \sqrt{1-\lambda}} Q(\mu)+\frac{\sqrt{1-\lambda}}{2 \sqrt{\lambda}} Q(\tau) .
$$

Integrating the above inequality over $\lambda \in(0,1)$ and replacing $\frac{\tau \mu}{\lambda \tau+(1-\lambda) \mu}$ by $x$ yield easily the right inequality of (5).

For any $\lambda \in(0,1)$, we have

$$
Q\left(\frac{2 \tau \mu}{\tau+\mu}\right) \leq \frac{1}{2}\left[Q\left(\frac{\tau \mu}{\lambda \mu+(1-\lambda) \tau}\right)+Q\left(\frac{\tau \mu}{\lambda \tau+(1-\lambda) \mu}\right)\right] .
$$

Integrating the above inequality with respect to $\lambda \in[0,1]$ gives

$$
Q\left(\frac{2 \tau \mu}{\tau+\mu}\right) \leq \frac{1}{2}\left[\int_{0}^{1} Q\left(\frac{\tau \mu}{\lambda \mu+(1-\lambda) \tau}\right) \mathrm{d} \lambda+\int_{0}^{1} Q\left(\frac{\tau \mu}{\lambda \tau+(1-\lambda) \mu}\right) \mathrm{d} \lambda\right] .
$$

Making use of the fact that

$$
\int_{0}^{1} Q\left(\frac{\tau \mu}{\lambda \mu+(1-\lambda) \tau}\right) \mathrm{d} \lambda=\int_{0}^{1} Q\left(\frac{\tau \mu}{\lambda \tau+(1-\lambda) \mu}\right) \mathrm{d} \lambda
$$

and replacing $\frac{\tau \mu}{\lambda \tau+(1-\lambda) \mu}$ by $x$ result in the left inequality of (5).

Multiplying both sides of inequality (6) by $2 \sqrt{t(1-t)}$ for $t \in(0,1)$ and simultaneously using the HT-convexity of $f$, we easily obtain the following corollaries.

Corollary 4.1 Suppose that $J \subseteq \mathbb{R} \backslash\{0\}$ is a nonempty interval, $Q \in \mathrm{HT}(J), \tau, \mu \in J$ with $\tau<\mu$, and $Q$ is Lebesgue integrable on $[\tau, \mu]$. Then

$$
Q\left(\frac{2}{\frac{1}{\mu}+\frac{1}{\tau}}\right) \leq \int_{0}^{1} Q\left(\frac{1}{\lambda \frac{1}{\tau}+(1-\lambda) \frac{1}{\mu}}\right) \mathrm{d} \lambda \leq \frac{\pi}{4}[Q(\tau)+Q(\mu)] .
$$


Corollary 4.2 Suppose that $J \subseteq \mathbb{R} \backslash\{0\}$ is a nonempty interval, $Q \in \mathrm{HT}(J), \tau, \mu \in J$ with $\tau<\mu$, and $Q$ is Lebesgue integrable on $[\tau, \mu]$. Then

$$
\frac{\pi}{4} Q\left(\frac{2 \tau \mu}{\tau+\mu}\right) \leq \frac{\tau \mu}{\mu-\tau} \int_{\tau}^{\mu} \tau(x) \frac{Q(x)}{x^{2}} \mathrm{~d} x \leq \frac{Q(\tau)+Q(\mu)}{2}
$$

where

$$
\tau(x)=2 \frac{\sqrt{\tau \mu(x-\tau)(\mu-x)}}{(\mu-\tau) x} .
$$

Corollary 4.3 Suppose that $J \subseteq \mathbb{R} \backslash\{0\}$ is a nonempty interval, $Q \in \mathrm{HT}(J), \tau, \mu \in J$ with $\tau<\mu$, and $Q$ is Lebesgue integrable on $[\tau, \mu]$. Then

$$
\frac{\pi}{2} Q\left(\frac{2 \tau \mu}{\tau+\mu}\right) \leq Q(\tau)+Q(\mu)
$$

Theorem 4.2 Suppose that $J \subseteq(0, \infty)$ is a nonempty interval, $Q \in \mathrm{HT}(J), \tau, \mu \in J$ with $\tau<\mu$, and $Q$ is Lebesgue integrable on $[\tau, \mu]$. Then, for $\lambda \in(0,1)$,

$$
\begin{aligned}
2 \sqrt{\lambda(1-\lambda)} Q\left(\frac{2 \tau \mu}{\tau+\mu}\right) \leq & (1-\lambda) Q\left(\frac{2 \tau \mu}{(1-\lambda) \tau+(1+\lambda) \mu}\right) \\
& +\lambda Q\left(\frac{2 \tau \mu}{(2-\lambda) \tau+\lambda \mu}\right) \\
\leq & \frac{\tau \mu}{\mu-\tau} \int_{\tau}^{\mu} \frac{Q(x)}{x^{2}} \mathrm{~d} x \\
\leq & \frac{\pi}{4}\left[(1-\lambda) Q(\tau)+\lambda Q(\mu)+Q\left(\frac{\tau \mu}{(1-\lambda) \tau+\lambda \mu}\right)\right] \\
\leq & \frac{\pi}{8 \sqrt{\lambda(1-\lambda)}}[Q(\tau)+Q(\mu)] .
\end{aligned}
$$

Proof Using inequality (7), for $\lambda \in(0,1)$, we have

$$
\begin{aligned}
Q\left(\frac{2 \tau \mu}{(1-\lambda) \tau+(1+\lambda) \mu}\right) & =Q\left(\frac{2}{(1-\lambda) \frac{1}{\mu}+\lambda \frac{1}{\tau}+\frac{1}{\tau}}\right) \\
& \leq \int_{0}^{1} Q\left(\frac{1}{(1-s)\left[(1-\lambda) \frac{1}{\mu}+\lambda \frac{1}{\tau}\right]+s \frac{1}{\tau}}\right) \mathrm{d} s \\
& =\int_{0}^{1} Q\left(\frac{\tau \mu}{(1-s)[(1-\lambda) \tau+\lambda \mu]+s \mu}\right) \mathrm{d} s \\
& \leq \frac{\pi}{4}\left[Q\left(\frac{\tau \mu}{(1-\lambda) \tau+\lambda \mu}\right)+Q(\tau)\right]
\end{aligned}
$$

and

$$
\begin{aligned}
Q\left(\frac{2 \tau \mu}{(2-\lambda) \tau+\lambda \mu}\right) & =Q\left(\frac{2}{\frac{1}{\mu}+(1-\lambda) \frac{1}{\mu}+\lambda \frac{1}{\tau}}\right) \\
& \leq \int_{0}^{1} Q\left(\frac{1}{(1-s) \frac{1}{\mu}+s\left[(1-\lambda) \frac{1}{\mu}+\lambda \frac{1}{\tau}\right]}\right) \mathrm{d} s
\end{aligned}
$$




$$
\begin{aligned}
& =\int_{0}^{1} Q\left(\frac{\tau \mu}{(1-s) \tau+s[(1-\lambda) \tau+\lambda \mu]}\right) d s \\
& \leq \frac{\pi}{4}\left[Q(\mu)+Q\left(\frac{\tau \mu}{(1-\lambda) \tau+\lambda \mu}\right)\right] .
\end{aligned}
$$

Multiplying both sides of (9) and (10) by $1-\lambda$ and $\lambda$, respectively, adding the obtained inequalities, and making use of Lemma 2.1, we arrive at

$$
\begin{aligned}
(1 & -\lambda) Q\left(\frac{2 \tau \mu}{(1-\lambda) \tau+(1+\lambda) \mu}\right)+\lambda Q\left(\frac{2 \tau \mu}{(2-\lambda) \tau+\lambda \mu}\right) \\
& \leq \int_{0}^{1} Q\left(\frac{\tau \mu}{(1-\lambda) \tau+\lambda \mu}\right) \mathrm{d} \lambda \\
& \leq \frac{\pi}{4}(1-\lambda)\left[Q\left(\frac{\tau \mu}{(1-\lambda) \tau+\lambda \mu}\right)+Q(\tau)\right]+\frac{\pi}{4} \lambda\left[Q(\mu)+Q\left(\frac{\tau \mu}{(1-\lambda) \tau+\lambda \mu}\right)\right] \\
& =\frac{\pi}{4}\left[(1-\lambda) Q(\tau)+\lambda Q(\mu)+Q\left(\frac{\tau \mu}{(1-\lambda) \tau+\lambda \mu}\right)\right],
\end{aligned}
$$

which proves the second and third inequalities in (9).

By the HT-convexity of $Q$, we have

$$
\begin{aligned}
& (1-\lambda) Q\left(\frac{2 \tau \mu}{(1-\lambda) \tau+(1+\lambda) \mu}\right)+\lambda Q\left(\frac{2 \tau \mu}{(2-\lambda) \tau+\lambda \mu}\right) \\
& =2 \sqrt{\lambda(1-\lambda)} \frac{\sqrt{1-\lambda}}{2 \sqrt{\lambda}} Q\left(\frac{2 \tau \mu}{(1-\lambda) \tau+(1+\lambda) \mu}\right) \\
& \quad+2 \sqrt{\lambda(1-\lambda)} \frac{\sqrt{\lambda}}{2 \sqrt{1-\lambda}} Q\left(\frac{2 \tau \mu}{(2-\lambda) \tau+\lambda \mu}\right) \\
& \geq 2 \sqrt{\lambda(1-\lambda)} Q\left(\frac{2 \tau \mu}{(1-\lambda)[(1-\lambda) \tau+(1+\lambda) \mu]+\lambda[(2-\lambda) \tau+\lambda \mu]}\right) \\
& =2 \sqrt{\lambda(1-\lambda)} Q\left(\frac{2 \tau \mu}{\tau+\mu}\right),
\end{aligned}
$$

which proves the first inequality in (10).

Similarly, we obtain

$$
\begin{aligned}
& \frac{\pi}{4}\left[(1-\lambda) Q(\tau)+\lambda Q(\mu)+Q\left(\frac{\tau \mu}{(1-\lambda) \tau+\lambda \mu}\right)\right] \\
& \quad \leq \frac{\pi}{4}\left[(1-\lambda) Q(\tau)+\lambda Q(\mu)+\frac{\sqrt{\lambda}}{2 \sqrt{1-\lambda}} Q(\tau)+\frac{\sqrt{1-\lambda}}{2 \sqrt{\lambda}} Q(\mu)\right] \\
& \quad \leq \frac{\pi}{4}\left(\frac{\sqrt{\lambda}}{2 \sqrt{1-\lambda}}+\frac{\sqrt{1-\lambda}}{2 \sqrt{\lambda}}\right)[Q(\tau)+Q(\mu)] \\
& \quad=\frac{\pi}{8 \sqrt{\lambda(1-\lambda)}}[Q(\tau)+Q(\mu)]
\end{aligned}
$$

which proves the last inequality in (10). 
Remark 4.1 With the assumptions of Theorem 4.2, taking $\lambda=\frac{1}{2}$ leads to

$$
\begin{aligned}
Q\left(\frac{2 \tau \mu}{\tau+\mu}\right) & \leq \frac{1}{2}\left[Q\left(\frac{4 \tau \mu}{\tau+3 \mu}\right)+Q\left(\frac{4 \tau \mu}{3 \tau+\mu}\right)\right] \leq \frac{\tau \mu}{\mu-\tau} \int_{\tau}^{\mu} \frac{Q(x)}{x^{2}} \mathrm{~d} x \\
& \leq \frac{\pi}{8}\left[Q(\tau)+Q(\mu)+2 Q\left(\frac{2 \tau \mu}{\tau+\mu}\right)\right] \leq \frac{\pi}{4}[Q(\tau)+Q(\mu)] .
\end{aligned}
$$

Theorem 4.3 Suppose that $J \subseteq \mathbb{R} \backslash\{0\}$ is a nonempty interval, $P, Q \in \mathrm{HT}(J), \tau, \mu \in J$ with $\tau<\mu$, and PQ is Lebesgue integrable on $[\tau, \mu]$. If $P, Q$ are nonnegative, then

$$
\frac{\tau \mu}{\mu-\tau} \int_{\tau}^{\mu} \frac{\tau^{2}(x)}{x^{4}} P(x) Q(x) \mathrm{d} x \leq \frac{2 M(\tau, \mu)+N(\tau, \mu)}{6}
$$

where $\tau(x)$ is defined in (8) and $M(\tau, \mu)$ and $N(\tau, \mu)$ are defined in (3).

Proof Because $P, Q \in \mathrm{HT}(J)$, for $\lambda \in(0,1)$, we have

$$
Q\left(\frac{\tau \mu}{\lambda \tau+(1-\lambda) \mu}\right) \leq \frac{\sqrt{\lambda}}{2 \sqrt{1-\lambda}} Q(\mu)+\frac{\sqrt{1-\lambda}}{2 \sqrt{\lambda}} Q(\tau)
$$

and

$$
P\left(\frac{\tau \mu}{\lambda \tau+(1-\lambda) \mu}\right) \leq \frac{\sqrt{\lambda}}{2 \sqrt{1-\lambda}} P(\mu)+\frac{\sqrt{1-\lambda}}{2 \sqrt{\lambda}} P(\tau)
$$

Because $P, Q$ are nonnegative, we obtain

$$
\begin{aligned}
& Q\left(\frac{\tau \mu}{\lambda \tau+(1-\lambda) \mu}\right) P\left(\frac{\tau \mu}{\lambda \tau+(1-\lambda) \mu}\right) \\
& \quad \leq \frac{\lambda Q(\mu) P(\mu)}{4(1-\lambda)}+\frac{(1-\lambda) Q(\tau) P(\tau)}{4 \lambda}+\frac{Q(\tau) P(\mu)+Q(\mu) P(\tau)}{4},
\end{aligned}
$$

that is,

$$
\begin{aligned}
& \lambda(1-\lambda) Q\left(\frac{\tau \mu}{\lambda \tau+(1-\lambda) \mu}\right) P\left(\frac{\tau \mu}{\lambda \tau+(1-\lambda) \mu}\right) \\
& \quad \leq \frac{\lambda^{2} Q(\mu) P(\mu)+(1-\lambda)^{2} Q(\tau) P(\tau)+\lambda(1-\lambda)[Q(\tau) P(\mu)+Q(\mu) P(\tau)]}{4} .
\end{aligned}
$$

Integrating the above inequality with respect to $\lambda \in[0,1]$ and replacing $\frac{\tau \mu}{\lambda \tau+(1-\lambda) \mu}$ by $x$ result in (11).

Remark 4.2 Choosing $\lambda=\frac{1}{2}$ in inequality (12), we obtain

$$
\begin{aligned}
& Q\left(\frac{2 \tau \mu}{\tau+\mu}\right) P\left(\frac{2 \tau \mu}{\tau+\mu}\right) \\
& \quad \leq \frac{Q(\tau) P(\tau)+Q(\mu) P(\mu)+Q(\tau) P(\mu)+Q(\mu) P(\tau)}{4} .
\end{aligned}
$$


Corollary 4.4 Under conditions of Theorem 4.3, if P, Q are similarly ordered, then

$$
\frac{\tau \mu}{\mu-\tau} \int_{\tau}^{\mu} \frac{\tau^{2}(x)}{x^{4}} P(x) Q(x) \mathrm{d} x \leq \frac{Q(\tau) P(\tau)+Q(\mu) P(\mu)}{2},
$$

where $\tau(x)$ is defined in (8).

Corollary 4.5 Under conditions of Theorem 4.3, if $P, Q$ are nonnegative, then

$$
\begin{aligned}
& \frac{\tau \mu P(\mu)}{\mu-\tau} \int_{\tau}^{\mu} \frac{\mu(x-\tau)}{x(\mu-\tau)} \frac{\tau(x) Q(x)}{x^{2}} \mathrm{~d} x+\frac{\tau \mu P(\tau)}{\mu-\tau} \int_{\tau}^{\mu} \frac{\tau(\mu-x)}{x(\mu-\tau)} \frac{\tau(x) Q(x)}{x^{2}} \mathrm{~d} x \\
& \quad+\frac{\tau \mu Q(\mu)}{\mu-\tau} \int_{\tau}^{\mu} \frac{\mu(x-\tau)}{x(\mu-\tau)} \frac{\tau(x) P(x)}{x^{2}} \mathrm{~d} x+\frac{\tau \mu Q(\tau)}{\mu-\tau} \int_{\tau}^{\mu} \frac{\tau(\mu-x)}{x(\mu-\tau)} \frac{\tau(x) P(x)}{x^{2}} \mathrm{~d} x \\
& \leq \frac{\tau \mu}{\mu-\tau} \int_{\tau}^{\mu} \frac{\tau^{2}(x)}{x^{4}} P(x) Q(x) \mathrm{d} x+\frac{2 M(\tau, \mu)+N(\tau, \mu)}{24},
\end{aligned}
$$

where $\tau(x)$ is defined in (8) and $M(\tau, \mu)$ and $N(\tau, \mu)$ are defined in (3).

Corollary 4.6 Under conditions of Corollary 4.5, if P, Q are similarly ordered, then

$$
\begin{aligned}
& \frac{\tau \mu P(\mu)}{\mu-\tau} \int_{\tau}^{\mu} \frac{\mu(x-\tau)}{x(\mu-\tau)} \frac{\tau(x) Q(x)}{x^{2}} \mathrm{~d} x+\frac{\tau \mu P(\tau)}{\mu-\tau} \int_{\tau}^{\mu} \frac{\tau(\mu-x)}{x(\mu-\tau)} \frac{\tau(x) Q(x)}{x^{2}} \mathrm{~d} x \\
& \quad+\frac{\tau \mu Q(\mu)}{\mu-\tau} \int_{\tau}^{\mu} \frac{\mu(x-\tau)}{x(\mu-\tau)} \frac{\tau(x) P(x)}{x^{2}} \mathrm{~d} x+\frac{\tau \mu Q(\tau)}{\mu-\tau} \int_{\tau}^{\mu} \frac{\tau(\mu-x)}{x(\mu-\tau)} \frac{\tau(x) P(x)}{x^{2}} \mathrm{~d} x \\
& \leq \frac{\tau \mu}{\mu-\tau} \int_{\tau}^{\mu} \frac{\tau^{2}(x)}{x^{4}} P(x) Q(x) \mathrm{d} x+\frac{Q(\tau) P(\tau)+Q(\mu) P(\mu)}{8},
\end{aligned}
$$

where $\tau(x)$ is defined in (8).

Theorem 4.4 Suppose that $J \subseteq \mathbb{R} \backslash\{0\}$ is a nonempty interval, $P, Q \in \mathrm{HT}(J), \tau, \mu \in J$ with $\tau<\mu$, and $P Q$ is Lebesgue integrable on $[\tau, \mu]$. If $P, Q$ are nonnegative, then

$$
Q\left(\frac{2 \tau \mu}{\tau+\mu}\right) P\left(\frac{2 \tau \mu}{\tau+\mu}\right) \leq \frac{\pi}{16}[M(\tau, \mu)+N(\tau, \mu)],
$$

where $M(\tau, \mu)$ and $N(\tau, \mu)$ are defined in (3).

Proof Because $P, Q$ are nonnegative and HT-convex, for $\lambda \in(0,1)$,

$$
\begin{aligned}
Q\left(\frac{2 \tau \mu}{\tau+\mu}\right) & =Q\left(\frac{2 \tau \mu}{\lambda \tau+(1-\lambda) \mu+(1-\lambda) \tau+\lambda \mu}\right) \\
& \leq \frac{1}{2}\left[Q\left(\frac{\tau \mu}{\lambda \tau+(1-\lambda) \mu}\right)+Q\left(\frac{\tau \mu}{(1-\lambda) \tau+\lambda \mu}\right)\right] \\
& \leq \frac{1}{2}\left(\frac{\sqrt{\lambda}}{2 \sqrt{1-\lambda}}+\frac{\sqrt{1-\lambda}}{2 \sqrt{\lambda}}\right)[Q(\tau)+Q(\mu)] .
\end{aligned}
$$

Similarly, we have

$$
P\left(\frac{2 \tau \mu}{\tau+\mu}\right) \leq \frac{1}{2}\left(\frac{\sqrt{\lambda}}{2 \sqrt{1-\lambda}}+\frac{\sqrt{1-\lambda}}{2 \sqrt{\lambda}}\right)[P(\tau)+P(\mu)] .
$$


Multiplying (13) and (14) reveals

$$
\begin{aligned}
Q\left(\frac{2 \tau \mu}{\tau+\mu}\right) P\left(\frac{2 \tau \mu}{\tau+\mu}\right) \leq & \frac{1}{16}\left(\frac{\sqrt{\lambda}}{\sqrt{1-\lambda}}+\frac{\sqrt{1-\lambda}}{\sqrt{\lambda}}\right)^{2} \\
& \times[Q(\tau)+Q(\mu)][P(\tau)+P(\mu)] \\
& =\frac{1}{16} \frac{1}{\lambda(1-\lambda)}[Q(\tau)+Q(\mu)][P(\tau)+P(\mu)] .
\end{aligned}
$$

Integrating inequality $(15)$ with respect to $\lambda \in(0,1)$ leads to the stated result.

Again using inequalities (13) and (14), we derive the following corollaries.

Corollary 4.7 Under conditions of Theorem 4.4, if P, Q are similarly ordered, then

$$
Q\left(\frac{2 \tau \mu}{\tau+\mu}\right) P\left(\frac{2 \tau \mu}{\tau+\mu}\right) \leq \frac{\pi}{8}[Q(\tau) P(\tau)+Q(\mu) P(\mu)]
$$

Corollary 4.8 Under conditions of Theorem 4.4, if P, $Q$ are nonnegative, then

$$
\begin{aligned}
& Q\left(\frac{2 \tau \mu}{\tau+\mu}\right)[P(\tau)+P(\mu)]+P\left(\frac{2 \tau \mu}{\tau+\mu}\right)[Q(\tau)+Q(\mu)] \\
& \quad \leq \frac{16}{3 \pi} Q\left(\frac{2 \tau \mu}{\tau+\mu}\right) P\left(\frac{2 \tau \mu}{\tau+\mu}\right)+\frac{2}{\pi}[Q(\tau)+Q(\mu)][P(\tau)+P(\mu)] .
\end{aligned}
$$

Corollary 4.9 Under conditions of Corollary 4.8, if $P, Q$ are similarly ordered, then

$$
\begin{aligned}
& Q\left(\frac{2 \tau \mu}{\tau+\mu}\right)[P(\tau)+P(\mu)]+P\left(\frac{2 \tau \mu}{\tau+\mu}\right)[Q(\tau)+Q(\mu)] \\
& \quad \leq \frac{16}{3 \pi} Q\left(\frac{2 \tau \mu}{\tau+\mu}\right) P\left(\frac{2 \tau \mu}{\tau+\mu}\right)+\frac{4}{\pi}[Q(\tau) P(\tau)+Q(\mu) P(\mu)] .
\end{aligned}
$$

\section{Applications to some special means}

In this section, we will consider applications of our newly-established results to the following special means.

For real numbers $\tau, \mu>0$, the arithmetic mean and the $p$-logarithmic mean are respectively defined $[26,29]$ by

$$
A=A(\tau, \mu)=\frac{\tau+\mu}{2}
$$

and

$$
L_{p}=L_{p}(\tau, \mu)= \begin{cases}{\left[\frac{\mu^{p+1}-\tau^{p+1}}{(p+1)(\mu-\tau)}\right]^{1 / p},} & \tau \neq \mu \\ \tau, & \tau=\mu\end{cases}
$$

For the HT-convex function $Q:(1, \infty) \rightarrow \mathbb{R}, Q(x)=\frac{1}{x^{p}}$ for $p \geq 1$, applying Theorem 4.2 and Corollary 4.1, we derive the following inequalities involving $A$ and $L_{p}$. 
Theorem 5.1 Let $1<\tau<\mu$ and $p \geq 1$. Then

$$
\frac{\pi}{4} A^{p}\left(\frac{1}{\tau}, \frac{1}{\mu}\right) \leq \frac{\pi}{4 \tau^{p}} F\left(-p, \frac{3}{2}, 3, \frac{\mu-\tau}{\mu}\right) \leq A\left(\frac{1}{\tau^{p+1}}, \frac{1}{\mu^{p+1}}\right)
$$

and

$$
\begin{aligned}
A^{p}\left(\frac{1}{\tau}, \frac{1}{\mu}\right) & \leq \frac{1}{2^{p+1}}\left[A^{p}\left(\frac{3}{\tau}, \frac{1}{\mu}\right)+A^{p}\left(\frac{1}{\tau}, \frac{3}{\mu}\right)\right] \leq L_{p}^{p}\left(\frac{1}{\tau}, \frac{1}{\mu}\right) \\
& \leq \frac{\pi}{4}\left[A\left(\frac{1}{\tau}, \frac{1}{\mu}\right)+A^{p}\left(\frac{1}{\tau}, \frac{1}{\mu}\right)\right] \leq \frac{\pi}{2} A\left(\frac{1}{\tau^{p+1}}, \frac{1}{\mu^{p+1}}\right),
\end{aligned}
$$

where $F(\alpha, \beta, \gamma, x)$ is the hypergeometric function which can be represented by

$$
F(\alpha, \beta, \gamma, x)=\frac{\Gamma(\gamma)}{\Gamma(\beta) \Gamma(\gamma-\beta)} \int_{0}^{1} \frac{t^{\beta-1}(1-t)^{\gamma-\beta-1}}{(1-x t)^{\alpha}} \mathrm{d} t .
$$

\section{Conclusions}

In [15] and [23], the HA- and MT-convexity were defined and some Hadamard-type inequalities were obtained. As a generalization of these two convexity notions a new notion of "HT-convex functions" is introduced in this paper, some Hadamard-type inequalities for the new class of HT-convex functions and for the product of any two HT-convex functions are established, and, as applications, some inequalities for the arithmetic mean and the $p$-logarithmic mean are derived.

\section{Acknowledgements}

The authors thank anonymous referees for their careful corrections to and valuable comments on the original version of this paper. The authors appreciate Professor Constantin P. Niculescu (University of Craiova, Romania) for his careful corrections to references related to the HA-convexity.

\section{Funding}

This work was supported in part by the PhD Research Foundation of Inner Mongolia University for Nationalities (Grant No. BS401 and No. BS402), the Science Research Foundation of Inner Mongolia University for Nationalities (Grant No. NMDYB1777), and the Natural Science Foundation of Inner Mongolia (Grant No. 2019MS01007), China.

Availability of data and materials

Not applicable.

\section{Competing interests}

The authors declare that they have no competing interests.

Authors' contributions

All authors contributed equally to the manuscript and read and approved the final manuscript.

\section{Author details}

${ }^{1}$ College of Mathematics, Inner Mongolia University for Nationalities, Tongliao, China. ${ }^{2}$ Institute of Mathematics, Henan Polytechnic University, Jiaozuo, China. ${ }^{3}$ School of Mathematical Sciences, Tianjin Polytechnic University, Tianjin, China.

\section{Publisher's Note}

Springer Nature remains neutral with regard to jurisdictional claims in published maps and institutional affiliations.

Received: 30 September 2019 Accepted: 29 December 2019 Published online: 08 January 2020

\section{References}

1. Anderson, G.D., Vamanamurthy, M.K., Vuorinen, M.: Generalized convexity and inequalities. J. Math. Anal. Appl. 335, 1294-1308 (2007). https://doi.org/10.1016/j.jmaa.2007.02.016

2. Beckenbach, E.F.: Convex functions. Bull. Am. Math. Soc. 54, 439-460 (1948). https://doi.org/10.1090/S0002-9904-1948-08994-7 
3. Chu, Y.-M., Khan, M.A., Khan, T.U., Ali, T.: Generalizations of Hermite-Hadamard type inequalities for MT-convex functions. J. Nonlinear Sci. Appl. 9, 4305-4316 (2016). https://doi.org/10.22436/jnsa.009.06.72

4. Dragomir, S.S.: Inequalities of Hermite-Hadamard type for HA-convex functions. Moroccan J. Pure Appl. Anal. 3 83-101 (2017). https://doi.org/10.1515/mjpaa-2017-0008

5. Hardy, G.H., Littlewood, J.E., Pólya, G.: Inequalities, 2nd edn. Cambridge University Press, Cambridge (1952)

6. Hermite, C.: Sur deux limites d'une intégrale définie. Mathesis 3, 82 (1883)

7. Hölder, O.: Über einen Mittelwertsatz. Nachr. Ges. Wiss. Gött., 38-47 (1889)

8. İşcan, I.: Hermite-Hadamard type inequalities for harmonically convex functions. Hacet. J. Math. Stat. 43, 935-942 (2014). https://doi.org/10.15672/HJMS.2014437519

9. Işcan, I., Wu, S.: Hermite-Hadamard type inequalities for harmonically convex functions via fractional integrals. Appl. Math. Comput. 238, 237-244 (2014). https://doi.org/10.1016/j.amc.2014.04.020

10. Jensen, J.L.W.V: Om konvexe Funktioner og Uligheder mellem Middelvaerdier. Nyt Tidsskr. Math. 16B, 49-69 (1905)

11. Liu, W.: Ostrowski type fractional integral inequalities for MT-convex functions. Miskolc Math. Notes 16, 249-256 (2015). https://doi.org/10.18514/mmn.2015.1131

12. Liu, W., Wen, W., Park, J.: Hermite-Hadamard type inequalities for MT-convex functions via classical integrals and fractional integrals. J. Nonlinear Sci. Appl. 9, 766-777 (2016). https://doi.org/10.22436/jnsa.009.03.05

13. Niculescu, C.P., Persson, L.-E.: Convex Functions and Their Applications: A Contemporary Approach, 2nd edn. CMS Books in Mathematics/Ouvrages de Mathématiques de la SMC. Springer, Cham (2018). https://doi.org/10.1007/978-3-319-78337-6

14. Noor, M.A., Noor, K.I., Awan, M.U.: Some inequalities for geometrically-arithmetically $h$-convex functions. Creative Math. Inform. 23, 91-98 (2014)

15. Pachpatte, B.G.: On some inequalities for convex functions. RGMIA Res. Rep. Collect. 6(Suppl.), Article ID 1 (2003). http://rgmia.org/v6(E).php

16. Park, J.: Hermite-Hadamard-like type inequalities for twice differentiable MT-convex functions. Appl. Math. Sci. 9, 5235-5250 (2015)

17. Pečarić, J., Proschan, F., Tong, Y.L.: Convex Functions, Partial Orderings, and Statistical Applications. Mathematics in Science and Engineering, vol. 187. Academic Press, San Diego (1992)

18. Skala, H.J.: On the characterization of certain similarly ordered super-additive functionals. Proc. Am. Math. Soc. 126, 1349-1353 (1998). https://doi.org/10.1090/S0002-9939-98-04702-9

19. Stolz, O.: Grunzüge der Differential und Integralrechnung, vol. 1. Teubner, Leipzig (1893)

20. Tunç, M., Subas, Y., Karabayir, I.: On some Hadamard type inequalities for MT-convex functions. Int. J. Open Probl. Comput. Sci. Math. 6, 101-113 (2013)

21. Tunç, M., Yildirim, H.: On MT-convexity (2012). arXiv:1205.5453

22. Wang, J.-Q., Guo, B.-N., Qi, F.: Generalizations and applications of Young's integral inequality by higher order derivatives. J. Inequal. Appl. 2019, Article ID 243 (2019). https://doi.org/10.1186/s13660-019-2196-2

23. Wang, S.-H.: Hermite-Hadamard type inequalities for operator convex functions on the co-ordinates. J. Nonlinear Sci. Appl. 10, 1116-1125 (2017). https://doi.org/10.22436/jnsa.010.03.22

24. Wang, S.-H., Qi, F.: Inequalities of Hermite-Hadamard type for convex functions which are $n$-times differentiable. Math. Inequal. Appl. 16, 1269-1278 (2013). https://doi.org/10.7153/mia-16-97

25. Wu, Y., Qi, F., Niu, D.-W.: Integral inequalities of Hermite-Hadamard type for the product of strongly logarithmically convex and other convex functions. Maejo Int. J. Sci. Technol. 9, 394-402 (2015)

26. Xi, B.-Y., Gao, D.-D., Zhang, T., Guo, B.-N., Qi, F.: Shannon type inequalities for Kapur's entropy. Mathematics 7(1), Article ID 22 (2019). https://doi.org/10.3390/math7010022

27. Xi, B.-Y., Qi, F.: Hermite-Hadamard type inequalities for geometrically r-convex functions. Studia Sci. Math. Hung. 51 530-546 (2014). https://doi.org/10.1556/SScMath.51.2014.4.1294

28. Xi, B.-Y., Qi, F.: Inequalities of Hermite-Hadamard type for extended s-convex functions and applications to means. J. Nonlinear Convex Anal. 16, 873-890 (2015)

29. Xi, B.-Y., Wu, Y., Shi, H.-N., Qi, F.: Generalizations of several inequalities related to multivariate geometric means. Mathematics 7(6), Article ID 552 (2019). https://doi.org/10.3390/math7060552

30. Yin, H.-P., Qi, F.: Hermite-Hadamard type inequalities for the product of $(\alpha, m)$-convex functions. J. Nonlinear Sci. Appl. 8, 231-236 (2015). https://doi.org/10.22436/jnsa.008.03.07

31. Yin, H.-P., Qi, F.: Hermite-Hadamard type inequalities for the product of $(\alpha, m)$-convex functions. Mo. J. Math. Sci. 27 71-79 (2015). http://projecteuclid.org/euclid.mjms/1449161369

32. Zhao, T.-H., Chu, Y.-M., Jiang, Y.-P.: Monotonic and logarithmically convex properties of a function involving gamma functions. J. Inequal. Appl. 2009, Article ID 728612 (2009). https://doi.org/10.1155/2009/728612

\section{Submit your manuscript to a SpringerOpen ${ }^{\circ}$ journal and benefit from:}

- Convenient online submission

- Rigorous peer review

- Open access: articles freely available online

- High visibility within the field

- Retaining the copyright to your article

Submit your next manuscript at $>$ springeropen.com 